\title{
ON THE SPECTRUM OF TOWERS
}

\author{
HAROLD DONNELLY \\ ABSTRACT. A new relation is derived between the spectrum of a noncompact \\ Riemannian manifold $M$ and certain sequences of compact quotients of $M$.
}

1. Introduction. Let $M$ be a complete Riemannian manifold with isometry group Iso $(M)$. A tower is a sequence of subgroups $\Gamma_{i} \subset \operatorname{Iso}(M), i \geqslant 1$, where each $\Gamma_{i}$ acts freely and properly discontinuously with compact quotient $\Gamma_{\mathrm{i}} \backslash M$. Of course, $\Gamma_{i} \backslash M$ is a compact Riemannian manifold. Moreover, we assume that the following requirements are satisfied: (a) for each $i, \Gamma_{i}$ is a normal subgroup of $\Gamma_{1}$, (b) $\bigcap_{i} \Gamma_{i}=1$, and (c) $\Gamma_{i+1}$ is of finite index in $\Gamma_{i}$. The last condition actually follows from the compactness of $\Gamma_{i} \backslash M$. We denote $\Gamma=\Gamma_{1}$.

For each $i$, the Laplacian $\Delta_{i}$ is a selfadjoint operator on $L^{2}\left(\Gamma_{i} \backslash M\right)$. Furthermore, $\Delta_{i}$ has a pure point spectrum consisting of discrete eigenvalues. Let $N_{i}(\lambda)$ be the number of eigenvalues, for $\Delta_{i}$, which are less than $\lambda$. The asymptotic behavior of $N_{i}(\lambda)$, as $\lambda \rightarrow \infty$, is an object of classical interest [2].

In this paper, we investigate the asymptotic growth of $N_{i}(\lambda)$ as $i \rightarrow \infty$, for fixed $\lambda$. The idea is to relate $N_{i}(\lambda)$, when $i \rightarrow \infty$, to the spectral theory of the noncompact manifold $M$. Since $M$ is complete, its Laplacian is essentially selfadjoint on $C_{0}^{\infty}(M)$. This means that $\Delta$ extends to a unique selfadjoint operator $\Delta: L^{2} M \rightarrow L^{2} M$.

Let $f \in C_{0}^{\infty}\left(R^{+}\right)$. For any $j$, the Laplacian $\Delta_{j}$ has pure point spectrum, since $\Gamma_{j} \backslash M$ is compact. Consequently, the operator $f\left(\Delta_{j}\right)$ has finite rank. In particular, $f\left(\Delta_{j}\right): L^{2}\left(\Gamma_{j} \backslash M\right) \rightarrow L^{2}\left(\Gamma_{j} \backslash M\right)$ is trace class.

Of course, since $M$ is noncompact, its Laplacian may have continuous spectrum. Consequently, $f(\Delta): L^{2} M \rightarrow L^{2} M$ need not be trace class in the usual sense. Nevertheless, we will show that $f(\Delta)$ is trace class in the von Neumann algebra of bounded operators on $L^{2} M$ which commute with $\Gamma$. Let $\operatorname{Tr}_{\Gamma}$ be the trace in this von Neumann algebra, as studied by M. F. Atiyah [1].

The main result of this paper is

THEOREM 1.1. For any $f \in C_{0}\left(R^{+}\right)$, a continuous function of compact support, one has

$$
\lim _{j \rightarrow \infty} \frac{\operatorname{Tr} f\left(\Delta_{j}\right)}{\operatorname{Vol}\left(\Gamma_{j} \backslash M\right)}=\frac{\operatorname{Tr}_{\Gamma} f(\Delta)}{\operatorname{Vol}(\Gamma \backslash M)} .
$$

Here $\operatorname{Vol}\left(\Gamma_{j} \backslash M\right)$ denotes the volume of $\Gamma_{j} \backslash M$. Recall that $\Gamma=\Gamma_{1}$.

Received by the editors August 3, 1981 and, in revised form. June 21, 1982

1980 Mathematics Subject Classification. Primary 53C99, 58G99.

'Supported in part by NSF Grant MCS 77-18723 A04. 
If $M$ admits a transitive group of isometries, then our results are well known $[5,6,11]$. In fact, strong theorems were proved for symmetric spaces by DeGeorge and Wallach [6]. These earlier papers inspired the present work.

2. The heat kernel. The main object of the present section is to provide the analytic machinery for the proof of our main theorem. However, the results obtained may have intrinsic interest.

We begin with

LEMMA 2.1. Suppose that $\Gamma_{j}, j \geqslant 1$, is a tower of subgroups, as defined in the Introduction. Set

$$
d_{j}=\inf _{\substack{\gamma \in \Gamma, j \\ \gamma \neq 1}} \inf _{\substack{x \in M \\ \gamma}} d(x, \gamma x)
$$

where $d$ is the geodesic distance on $M$. Then $d_{j} \rightarrow \infty$, as $j \rightarrow \infty$.

Proof. Since $\Gamma \backslash M$ is compact, there is a compact set $B$ in $M$ with $\Gamma B=M$. For convenience, we may suppose that $B$ is a ball of radius $r$, about some point $p \in M$. As usual, we employ the notation $\Gamma=\Gamma_{1}$.

Recall that each $\Gamma_{i}$ is a normal subgroup of $\Gamma$. Therefore, we may write

$$
d_{j}=\inf _{\substack{\gamma \in \Gamma, \gamma \neq 1}} \inf _{\substack{x \in M \\ \gamma}} d(x, \gamma x)=\inf _{\substack{\gamma \in \Gamma_{j} \\ \gamma \neq 1}} \inf _{x \in B} d(x, \gamma x) .
$$

We now reason by contradiction. If our conclusion fails, then there is a sequence $\gamma_{j} \in \Gamma_{j}$, so that $d\left(p, \gamma_{j} p\right)$ is bounded. More specifically, $d\left(p, \gamma_{j} p\right)<r^{\prime}$ for some $r^{\prime}$ and all $j$.

Let $B^{\prime}$ be the ball of radius $r^{\prime}$ about $p$. Then $B^{\prime}$ intersects an infinite number of its $\Gamma$ translates. This contradicts the fact that $\Gamma$ acts properly discontinuously.

In the author's earlier paper [7], the main objects of study were the operators $\exp (-t \Delta)$ and $\exp \left(-t \Delta_{j}\right)$, for $t>0$. These fundamental solutions of the heat equation problem are given by smoothing kernels,

$$
\exp (-t \Delta) g(x)=\int_{M} \exp (-t \Delta)(x, y) g(y) d y
$$

and

$$
\exp \left(-t \Delta_{j}\right) h(x)=\int_{\Gamma, M} \exp \left(-t \Delta_{j}\right)(x, y) h(y) d y
$$

for $g \in L^{2} M$ and $h \in L^{2}\left(\Gamma_{j} \backslash M\right)$. The kernels appearing äre $C^{\infty}$ functions for $t>0$ and arbitrary $x, y$.

The basic information needed now is contained in

LEMMA 2.2. For any $t>0$, we may write

$$
\exp \left(-t \Delta_{j}\right)(x, x)=\exp (-t \Delta)(x, x)+O\left(e^{-c_{j} / t}\right) .
$$

Here, the constant $c_{j} \rightarrow \infty$ as $j \rightarrow \infty$. This estimate is uniform for $t$ in a compact subinterval of $(0, \infty)$. 
Proof. Theorem 4.3 of [7] states that $\exp \left(-t \Delta_{j}\right)(x, y)=\Sigma_{\gamma \in \Gamma,} \exp (-t \Delta)(x, \gamma y)$ in the sense of uniform convergence.

In particular, for $x=y$, one has

$$
\exp \left(-t \Delta_{j}\right)(x, x)=\exp (-t \Delta)(x, x)+\sum_{\substack{\gamma \in \Gamma_{,} \\ \gamma \neq 1}} \exp (-t \Delta)(x, \gamma x) \text {. }
$$

The main estimate of $[7$, p. 491] gives

$$
\sum_{\substack{\gamma \in \Gamma_{1} \\ \gamma \neq 1}} \exp (-t \Delta)(x, \gamma x)=O\left(e^{-\alpha d_{i}^{2} / t}\right) .
$$

Here $\alpha$ is a fixed constant, independent of $j$. Moreover, $d_{j}$ is as given in Lemma 2.1.

Set $c_{j}=\alpha d_{j}^{2}$. The result now follows from Lemma 2.1.

By isometry invariance of the heat kernel, one has, for $x \in M$ and $\gamma \in \Gamma$, $\exp (-t \Delta)(x, x)=\exp (-t \Delta)(\gamma x, \gamma x)$. Thus $\exp (-t \Delta)(x, x)$ may be considered as a function on $\Gamma \backslash M$.

The main result of this section is

THEOREM 2.3. For any fixed $t>0$, one has

$$
\lim _{j \rightarrow \infty} \frac{\operatorname{Tr}\left(e^{-t \Delta,}\right)}{\operatorname{Vol}\left(\Gamma_{j} \backslash M\right)}=\frac{\int_{\Gamma \backslash M} e^{-t \Delta}(x, x) d x}{\operatorname{Vol}(\Gamma \backslash M)}=\frac{\operatorname{Tr}_{\Gamma} e^{-t \Delta}}{\operatorname{Vol}(\Gamma \backslash M)}
$$

Here Vol denotes the volume. Moreover, the convergence is uniform for $t$ in a compact subinterval of $(0, \infty)$.

Proof. The second equality, and the fact that $\exp (-t \Delta)$ is $\Gamma$-trace class follow from [1, pp. 63-64].

To prove the first equality, we write

$$
\frac{\operatorname{Tr}\left(e^{-t \Delta}\right)}{\operatorname{Vol}\left(\Gamma_{j} \backslash M\right)}=\frac{\int_{\Gamma, M} e^{-t \Delta,}(x, x) d x}{\operatorname{Vol}\left(\Gamma_{j} \backslash M\right)}
$$

By Lemma 2.2, one has

$$
\frac{\operatorname{Tr}\left(e^{-t \Delta_{1}}\right)}{\operatorname{Vol}\left(\Gamma_{j} \backslash M\right)}=\frac{\int_{\Gamma_{,}, M} e^{-t \Delta}(x, x) d x}{\operatorname{Vol}\left(\Gamma_{j} \backslash M\right)}+O\left(e^{-c_{1} / t}\right)
$$

However, using the isometry invariance of the heat kernel, we have

$$
\frac{\operatorname{Tr}\left(e^{-t \Delta,}\right)}{\operatorname{Vol}\left(\Gamma_{j} \backslash M\right)}=\frac{\int_{\Gamma \backslash M} e^{-t \Delta}(x, x)}{\operatorname{Vol}(\Gamma \backslash M)}+O\left(e^{-c, / t}\right)
$$

Recall that $\Gamma=\Gamma_{1}$ and each $\Gamma_{j}$ is a normal subgroup of $\Gamma$.

Letting $j \rightarrow \infty$, we obtain Theorem 2.3.

By using known estimates of the heat kernel, for manifolds $M$ with bounded geometry, one deduces 
COROLlaRY 2.4. Suppose that $M$ is simply connected and has nonpositive curvature. Then, for each fixed $\lambda>0$, we may write

$$
\varlimsup_{j \rightarrow \infty} \frac{N_{j}(\lambda)}{\operatorname{Vol}\left(\Gamma_{j} \backslash M\right)} \leqslant(4 \pi)^{-n / 2} e \lambda^{n / 2} .
$$

Here $n$ is the dimension of $M$.

Proof. For any $t>0$ and $\alpha>0$, one has, by Theorem 2.3,

$$
\lim _{j \rightarrow \infty} \frac{\alpha \operatorname{Tr}\left(e^{-t \Delta,}\right)}{\operatorname{Vol}\left(\Gamma_{j} \backslash M\right)}=\frac{\alpha \int_{\Gamma \backslash M} e^{-t \Delta}(x, x) d x}{\operatorname{Vol}(\Gamma \backslash M)} .
$$

By comparison with the Eucliden heat kernel [9], it follows that $\exp (-t \Delta)(x, x) \leqslant$ $(4 \pi t)^{-n / 2}$. To do this comparison, one needs that $M$ is complete, simply connected, and of nonpositive curvature.

Thus

$$
\lim _{j \rightarrow \infty} \frac{\alpha \operatorname{Tr}\left(e^{-t \Delta,}\right)}{\operatorname{Vol}\left(\Gamma_{j} \backslash M\right)} \leqslant(4 \pi t)^{-n / 2} \alpha .
$$

Now choose $\alpha=e^{t \lambda}$, so that $\alpha e^{-t y}>1$ for $y<\lambda$.

Then, for any $t>0$,

$$
\varlimsup_{j \rightarrow \infty} \frac{N_{j}(\lambda)}{\operatorname{Vol}\left(\Gamma_{j} \backslash M\right)} \leqslant \lim _{j \rightarrow \infty} \frac{\alpha \operatorname{Tr}\left(e^{-t \Delta}\right)}{\operatorname{Vol}\left(\Gamma_{j} \backslash M\right)} \leqslant(4 \pi t)^{-n / 2} e^{t \lambda} .
$$

Corollary 2.4 follows by choosing $t=1 / \lambda$.

Similarly, one may write

COROLlaRy 2.5. Let $C_{1}$ be a lower bound for the injectivity radius of $M$, and $C_{2}$ an upper bound on the absolute value of the sectional curvature of $M$. Then

$$
\varlimsup_{j \rightarrow \infty} \frac{N_{j}(\lambda)}{\operatorname{Vol}\left(\Gamma_{j} \backslash M\right)} \leqslant C_{3} \lambda^{n / 2} .
$$

Here, $C_{3}$ depends only upon $C_{1}$ and $C_{2}$. Moreover, $n$ is the dimension of $M$.

Proof. Under the given hypotheses, it is well known $[3,4$ and 8] that

$$
\exp (-t \Delta)(x, x) \leqslant C_{4} t^{-n / 2} \text {. }
$$

The constant $C_{4}$ depends only upon $C_{1}$ and $C_{2}$.

Given this estimate, one follows the proof of Corollary 2.4, with small modifications.

To see that these upper bounds have the correct dependence upon $\lambda$, it is useful to consider an example. Let $\Gamma_{j} \backslash M$ be an $n$-torus. More specifically, $\Gamma_{j} \backslash M=S_{j} \times S_{j}$ $\times \cdots \times S_{j}$, where $S_{j}$ is a circle of circumference $j$. For this example, it follows by the geometry of numbers that

$$
\lim _{j \rightarrow \infty} \frac{N_{j}(\lambda)}{\operatorname{Vol}\left(\Gamma_{j} \backslash M\right)}=(4 \pi)^{-n / 2} \frac{\lambda^{n / 2}}{\Gamma(n / 2+1)} .
$$

Note that this is compatible with Corollaries 2.4 and 2.5. 
3. Proof of Theorem 1.1. The proof of our main theorem follows, by an approximation argument, from Theorem 2.3.

In preparation, we recall the elementary lemma,

Lemma 3.1. Let $f \in C_{0}\left(R^{+}\right)$. Then $f$ may be uniformly approximated by finite linear combinations of exponentials $\exp (-t x), t>0$.

Proof. Suppose that $0<x<\infty$ and make the change of variables $y=e^{-x}$, $0<y<1$. Define $g(y)=f(x)$. Then $g \in C_{0}((0,1])$.

Given $\varepsilon>0$, the Weierstrass approximation theorem gives a polynomial close to g,

$$
\left|g(y)-\sum_{i=0}^{n} a_{i} y^{i}\right|<\varepsilon / 2 .
$$

Since $g(0)=0$, it follows that $\left|a_{0}\right|<\varepsilon / 2$. Thus $\left|g(y)-\sum_{i=1}^{n} a_{i} y^{\prime}\right|<\varepsilon$.

Changing back to the variable $x$ gives the required estimate $\left|f(x)-\sum_{i=1}^{n} a_{i} e^{-i x}\right|<$ $\varepsilon$.

We turn to the proof of Theorem 1.1.

If $f \in C_{0}\left(R^{+}\right)$, then define, for $x \in R^{+}, g(x)=f(x) e^{x}$. Of course, $g \in C_{0}\left(R^{+}\right)$. Now $g(\Delta)$ is bounded on $L^{2} M$. Also, by Theorem 2.3, $e^{-\Delta}$ is of trace class in the von Neumann algebra of bounded operators commuting with $\Gamma$. According to [1, p. 59], the trace class operators form a two-sided ideal in this von Neumann algebra. Consequently, $f(\Delta)=g(\Delta) e^{-\Delta}$ is $\Gamma$-trace class.

Using Lemma 3.1, we may choose a sequence $g_{k}(x)$, of polynomials in $e^{-x}$. which uniformly approximate $g(x)$. Then, one has for any $j$,

$$
\left|\frac{\operatorname{Tr}_{\Gamma} f(\Delta)}{\operatorname{Vol}(\Gamma \backslash M)}-\frac{\operatorname{Tr} f(\Delta)}{\operatorname{Vol}\left(\Gamma_{j} \backslash M\right)}\right| \leqslant \mathscr{D}_{1}+\mathrm{OD}_{2}+\mathrm{DQ}_{3}
$$

where

$$
\begin{aligned}
& \mathscr{Q}_{1}=\left|\frac{\operatorname{Tr}_{\Gamma} g(\Delta) e^{-\Delta}}{\operatorname{Vol}(\Gamma \backslash M)}-\frac{\operatorname{Tr}_{\Gamma} g_{k}(\Delta) e^{-\Delta}}{\operatorname{Vol}(\Gamma \backslash M)}\right|, \\
& \mathscr{D}_{2}=\left|\frac{\operatorname{Tr}_{\Gamma} g_{k}(\Delta) e^{-\Delta}}{\operatorname{Vol}(\Gamma \backslash M)}-\frac{\operatorname{Tr} g_{k}\left(\Delta_{j}\right) e^{-\Delta}}{\operatorname{Vol}\left(\Gamma_{j} \backslash M\right)}\right|
\end{aligned}
$$

and

$$
\mathscr{D}_{3}=\left|\frac{\operatorname{Tr} g_{k}\left(\Delta_{j}\right) e^{-\Delta}}{\operatorname{Vol}\left(\Gamma_{j} \backslash M\right)}-\frac{\operatorname{Tr} g\left(\Delta_{j}\right) e^{-\Delta_{j}}}{\operatorname{Vol}\left(\Gamma_{j} \backslash M\right)}\right| .
$$

The spectral theorem for selfadjoint operators implies that $g_{k}(\Delta)$ converge strongly to $g(\Delta)$. Therefore, by employing [1, p. 63], we may choose $k$ sufficiently large so that $\mathscr{D}_{1} \leqslant \varepsilon / 3$. 
To estimate $\mathscr{D}_{3}$, note that

$$
\mathscr{D}_{3} \leqslant \sup _{x \in R^{+}}\left|g_{k}(x)-g(x)\right|\left|\frac{\operatorname{Tr}\left(e^{-\Delta_{j}}\right)}{\operatorname{Vol}\left(\Gamma_{j} \backslash M\right)}\right| \leqslant C_{1} \sup _{x \in R^{+}}\left|g_{k}(x)-g(x)\right|
$$

by Theorem 2.3 . Here $C_{1}$ is a positive constant. So, by choosing $k$ sufficiently large, we have $\mathscr{D}_{3} \leqslant \varepsilon / 3$, for all $j$.

Now, fix $k$ so that $\mathscr{D}_{1}+\mathscr{D}_{3} \leqslant 2 \varepsilon / 3$. By Theorem 2.3 , we have $\mathscr{D}_{2} \leqslant \varepsilon / 3$, for $j$ sufficiently large.

In conclusion, we have shown that

$$
\left|\frac{\operatorname{Tr}_{\Gamma} f(\Delta)}{\operatorname{Vol}(\Gamma \backslash M)}-\frac{\operatorname{Tr} f\left(\Delta_{j}\right)}{\operatorname{Vol}\left(\Gamma_{j} \backslash M\right)}\right| \leqslant \varepsilon
$$

for $j$ sufficiently large. This proves Theorem 1.1 .

4. Applications of Theorem 1.1. The main use for Theorem 1.1 is that one can employ information about the spectrum of $\Delta$ to deduce facts concerning the spectrum of $\Delta_{j}$, for $j$ suitably large. In many cases, it is easier to investigate the Laplacian of the noncompact manifold $M$ than to study directly the Laplacian on the compact quotients $\Gamma_{j} \backslash M$.

Let $N_{j}(\lambda)$ denote the number of eigenvalues of $\Delta$, which are less than $\lambda$. Suppose one lets $\lambda_{1}(M)$ be the greatest lower bound for the Laplacian on $M$. By the minimax principle,

$$
\lambda_{1}(M)=\inf _{f \in C_{0}^{\infty} M} \frac{\langle d f, d f\rangle_{2}}{\langle f, f\rangle_{2}} .
$$

A basic consequence of Theorem 1.1 is

Corollary 4.2. (i) If $\lambda<\lambda_{1}(M)$, then

$$
\limsup _{j \rightarrow \infty} \frac{N_{j}(\lambda)}{\operatorname{Vol}\left(\Gamma_{j} \backslash M\right)}=0
$$

(ii) If $\lambda>\lambda_{1}(M)$, then

$$
\liminf _{j \rightarrow \infty} \frac{N_{j}(\lambda)}{\operatorname{Vol}\left(\Gamma_{j} \backslash M\right)}>0 .
$$

Proof. (i) Let $f \in C_{0}\left(\left[0, \lambda_{1}(M)\right]\right)$ be equal to one on the interval $[0, \lambda)$. Then, for any $j$, one has $N_{j}(\lambda)<\operatorname{Tr} f\left(\Delta_{j}\right)$. Since $f(\Delta)=0$, Theorem 1.1 gives

$$
\limsup _{j \rightarrow \infty} \frac{N_{j}(\lambda)}{\operatorname{Vol}\left(\Gamma_{j} \backslash M\right)} \leqslant \lim _{j \rightarrow \infty} \frac{\operatorname{Tr} f(\Delta)}{\operatorname{Vol}\left(\Gamma_{j} \backslash M\right)}=0 .
$$

(ii) This is an elementary result which follows easily from Dirichlet bracketing. Denote $\bar{\Delta}_{j}$ to be the Laplacian on a fundamental domain for $\Gamma_{j}$, with Dirichlet boundary conditions. Let $\bar{N}_{j}(\lambda)$ be the number of eigenvalues of $\bar{\Delta}$ less than $\lambda$.

The minimax principle gives the inequality $N_{j}(\lambda) \geqslant \bar{N}_{j}(\lambda)$, for each $j$. Similarly, from (4.1), one has $\bar{N}_{j}(\lambda)>0$ for $j$ sufficiently large, since $\lambda>\lambda_{1}(M)$. 
However, translating by a finite set of coset representatives for $\Gamma_{k} \subset \Gamma_{j}, k \geqslant j$, the isometry invariance of $\Delta$ gives

$$
\frac{\bar{N}_{j}(\lambda)}{\operatorname{Vol}\left(\Gamma_{j} \backslash M\right)} \leqslant \frac{\bar{N}_{k}(\lambda)}{\operatorname{Vol}\left(\Gamma_{k} \backslash M\right)}
$$

So this ratio increases as the index $j$ increases.

Assembling these facts, one has

$$
\liminf _{j \rightarrow \infty} \frac{N_{j}(\lambda)}{\operatorname{Vol}\left(\Gamma_{j} \backslash M\right)} \geqslant \liminf _{i \rightarrow \infty} \frac{\bar{N}_{k+i}(\lambda)}{\operatorname{Vol}\left(\Gamma_{k+i} \backslash M\right)} \geqslant \frac{\bar{N}_{k}(\lambda)}{\operatorname{Vol}\left(\Gamma_{k} \backslash M\right)}
$$

for any $k$. Since the right-hand side is positive for $k$ sufficiently large, the proof of Corollary 4.2 is complete.

If $M$ has variable negative curvature bounded above by $-c^{2}$, then $\lambda_{1}(M) \geqslant$ $(d-1)^{2} c^{2} / 4$, where $d$ is the dimension of $M$ [12]. Thus, one may apply Corollary 4.2(i) to deduce that

$$
\limsup _{j \rightarrow \infty} \frac{N_{j}(\lambda)}{\operatorname{Vol}\left(\Gamma_{j} \backslash M\right)}=0
$$

for $\lambda<(d-1)^{2} c^{2} / 4$.

Another interesting extension of Theorem 1.1 is

COROLlaRY 4.3. Suppose that the Laplacian $\Delta$ of $M$ has no point spectrum. Then

$$
\lim _{j \rightarrow \infty} \frac{\operatorname{Tr} h\left(\Delta_{j}\right)}{\operatorname{Vol}\left(\Gamma_{j} \backslash M\right)}=\frac{\operatorname{Tr}_{\Gamma} h(\Delta)}{\operatorname{Vol}(\Gamma \backslash M)}
$$

where $h$ is the characteristic function of any bounded interval in $R^{+}$.

Proof. Let $h$ be the characteristic function of the interval $\left[\lambda_{1}, \lambda_{2}\right]$. Choose continuous functions $f_{k}, g_{k}$ satisfying the following conditions: (i) $0 \leqslant f_{k} \leqslant h \leqslant g_{k} \leqslant$ 1, and (ii) $f_{k}(x)=h(x)=g_{k}(x)$ unless $\left|x-\lambda_{1}\right|<1 / k$ or $\left|x-\lambda_{2}\right|<1 / k$.

Clearly, $\operatorname{Tr} f_{k}\left(\Delta_{j}\right) \leqslant \operatorname{Tr} h\left(\Delta_{j}\right) \leqslant \operatorname{Tr} g_{k}\left(\Delta_{j}\right)$ for each $j$ and $k$.

Therefore, by Theorem 1.1, we have

$$
\frac{\operatorname{Tr}_{\Gamma} f_{k}(\Delta)}{\operatorname{Vol}(\Gamma \backslash M)} \leqslant \liminf _{j \rightarrow \infty} \frac{\operatorname{Tr} h\left(\Delta_{j}\right)}{\operatorname{Vol}\left(\Gamma_{j} \backslash M\right)} \leqslant \limsup _{j \rightarrow \infty} \frac{\operatorname{Tr} h\left(\Delta_{j}\right)}{\operatorname{Vol}\left(\Gamma_{j} \backslash M\right)} \leqslant \frac{\operatorname{Tr}_{\Gamma} g_{k}(\Delta)}{\operatorname{Vol}(\Gamma \backslash M)}
$$

for any $k$.

Set $\bar{f}_{k}(x)=f(x) e^{x}, \bar{g}_{k}(x)=g(x) e^{x}$, and $\bar{h}(x)=h(x) e^{x}$. Then, the spectral theory for selfadjoint operators gives that $\bar{f}_{k}(\Delta)$ and $\bar{g}_{k}(\Delta)$ converge strongly to $\bar{h}(\Delta)$. Here, we use the fact that $\Delta$ has no point spectrum.

Given this strong convergence, we may apply [1, p. 63] to write

$$
\lim _{k \rightarrow \infty} \operatorname{Tr}_{\Gamma} f_{k}(\Delta)=\lim _{k \rightarrow \infty} \operatorname{Tr}_{\Gamma} \bar{f}_{k}(\Delta) e^{-\Delta}=\operatorname{Tr}_{\Gamma} \bar{h}(\Delta) e^{-\Delta}=\operatorname{Tr}_{\Gamma} h(\Delta) .
$$

In particular, the factorization $h(\Delta)=\bar{h}(\Delta) e^{-\Delta}$ shows that $h(\Delta)$ is of $\Gamma$-trace class [1, p. 59]. Similarly, $\operatorname{Tr}_{\Gamma} g_{k}(\Delta)$ converges to $\operatorname{Tr}_{\Gamma} h(\Delta)$ as $k \rightarrow \infty$.

Corollary 4.3 now follows by referring to (4.4). 
If $M$ is a symmetric space of noncompact type, then $\Delta$ has no point spectrum on functions in $L^{2} M$ [10]. In this case, Corollary 4.3 was proved by DeGeorge and Wallach [6].

\section{BIBLIOGRAPHY}

I. M. F. Atiyah, Elliptic operators, discrete groups, and von Neumann algebras, Astérisque 32-33 (1976), 43-72.

2. M. Berger, P. Gauduchon and E. Mazet, Le spectre d'une varieté Riemannienne. Springer-Verlag. Berlin. Heidelberg and New York, 1971.

3. J. Cheeger, M. Gromov and M. Taylor, Finite propogation speed, kernel estimates for functions of the Laplace operator, and the geometry of complete Riemannian manifolds, J. Differential Geometry 17 (1982), $15-54$.

4. S. Y. Cheng, P. Li and S. T. Yau, On the upper estimate of the heat kernel of a complete Riemannian manifold, Amer. J. Math. 103 (1981), 1021-1063.

5. L. Corwin. The Plancherel measure in nilpotent Lie groups as a limit of point measures, Math. Z. 155 (1977). 151-162.

6. D. DeGeorge and N. Wallach, Limit formulas for multiplicities in $L^{2}(\Gamma \backslash G)$. Ann. of Math. (2) 107 (1978), 133-150.

7. H. Donnelly, Asymptotic expansions for the compact quotients of properly discontinuous group actions, Illinois J. Math. 23 (1979), 485-496.

8. Stability theorems for the continuous spectrum of a negatively curved manifold. Trans. Amer. Math. Soc. 264 (1981), 431-448.

9. H. Donnelly and P. Li, Lower hounds for the eigenvalues of negatively curved manifolds, Math. Z. 172 (1980), 29-40.

10. S. Helgason, Functions on symmetric spaces, Proc. Sympos. Pure Math., vol. 26, Amer. Math. Soc., Providence, R.I., 1973, pp. 101-146.

11. H. Huber, Üher die Eigenwerte des Laplace Operators auf kompakten Riemannschen Flächen. Comment. Math. Helv. 51 (1976), 215-231.

12. S. T. Yau, Isoperimetric constants and the first eigenvalue of a compact Riemannian manifold, Ann. Sci. Ecole Norm. Sup. (4) 8 (1975), 487-507.

Department of Mathematics, Purdue University, West Lafayette, Indiana 47907 